\title{
Security of Invertible Media Authentication Schemes Revisited
}

\author{
Daniel Dönigus, Stefan Endler, Marc Fischlin, Andreas Hülsing, Patrick Jäger, \\ Anja Lehmann, Sergey Podrazhansky, Sebastian Schipp, Erik Tews, \\ Sven Vowe, Matthias Walthart, and Frederik Weidemann \\ Darmstadt University of Technology, Germany \\ marc.fischlin@gmail.com, \\ www.minicrypt.de
}

\begin{abstract}
Dittmann, Katzenbeisser, Schallhart and Veith (SEC 2005) introduced the notion of invertible media authentication schemes, embedding authentication data in media objects via invertible watermarks. These invertible watermarks allow to recover the original media object (given a secret encryption key), as required for example in some medical applications where the distortion must be removable.

Here we revisit the approach of Dittmann et al. from a cryptographic viewpoint, clarifying some important aspects of their security definitions. Namely, we first discuss that their notion of unforgeability may not suffice in all settings, and we therefore propose a strictly stronger notion. We then show that the basic scheme suggested by Dittmann et al. achieves our notion if instantiated with the right cryptographic primitives. Our proof also repairs a flaw in the original scheme, pointed out by Hopper, Molnar and Wagner (TCC 2007).

We finally address the issue of secrecy of media authentication schemes, basically preventing unauthorized recovering of the original media object without the encryption key. We give a rigorous security statement (that is, the best security guarantee we can achieve) and prove again that the scheme by Dittmann et al. meets this security level if the right cryptographic building blocks are deployed. Together our notions of unforgeability and of secrecy therefore give very strong security guarantees for such media authentication schemes.
\end{abstract}

\section{Introduction}

The transition from analog to digital media facilitates many tasks but also comes along with continually improved manipulation tools, which allow various modifications of media objects. Thus, it becomes increasingly difficult to distinguish authentic from altered objects. To enable a better distinction it is therefore necessary to apply techniques that guarantee authenticity, integrity and possibly secrecy of data.

The straightforward use of digital signatures is not always a satisfying solution to provide authenticity and integrity, because an object and its signature have to be stored separately. This, however, may not be convenient in the area 
of multimedia data. To counter this problem fragile watermarks were proposed, which can be used to embed a signature directly into an object, such that any (significant) modification will destroy the watermark and thereby invalidates the signature. Unfortunately, this approach comes with the disadvantage that it always leads to irrevocable distortions in the authenticated object, which may not be acceptable in all applications, e.g., X-ray imaging objects are extremely sensitive to modifications. One solution is to use invertible watermarking schemes, which are special fragile watermarks addressing the need to re-obtain the original media object by allowing a complete removal of the embedded data.

Media Authentication Schemes. Using invertible watermarking schemes in combination with encryption and digital signatures, Dittmann, Katzenbeisser, Schallhart and Veith (DKSV) 3] introduced the notion of an invertible media authentication scheme that allows reconstruction of the original object. They also propose a framework to build such authentication schemes: To protect a media object $O$ the $\mathcal{M} \mathcal{A S}_{\text {DKSV }}$ scheme first applies an invertible watermarking scheme as proposed by Fridrich et al. 4, dividing $O$ into two parts $A_{O}, B_{O}$ by running the watermarking algorithm SEPARATE. See Figure 1 The part $B_{O}$ next gets compressed and encrypted to a ciphertext $X$ that is stored as the first part of the watermark. To achieve an appropriate compressibility level, $B_{O}$ has to be chosen accordingly. The second part of the watermark contains the digital signature $s$ of the encrypted part $X$ and $A_{O}$, the public part of the object. Finally, the watermark $(X, s)$ is joined with $A_{O}$ to a single protected object $\bar{O}$ by using the watermarking algorithm JoIN.

Reconstruction of the original object from $\bar{O}$ is done by decrypting to recover $\operatorname{Compress}\left(B_{O}\right)$ and uncompressing this value to get the part $B_{O}$. A simple join operation with $A_{O}$ merges the parts together again. As for integrity and secrecy, as long as the object is not altered the signature can be verified by using the public verification key, while the reconstruction of the original object is protected by the secret reconstruction key.

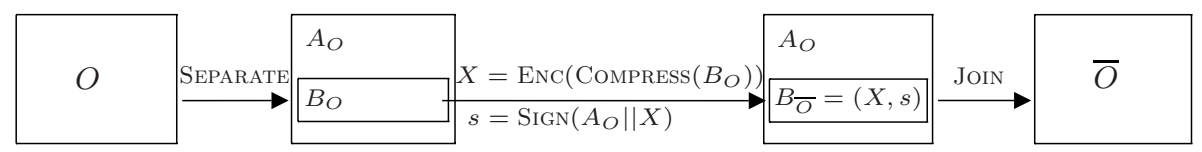

Fig. 1. Protection of media objects in the $\mathcal{M} \mathcal{A} \mathcal{S}_{\mathrm{DKSV}}$ Scheme

In contrast to most known watermarking schemes where the security is only analyzed by ad-hoc methods, the media authentication scheme of Dittmann et al. comes with a formal model against malicious modification attempts, following well-known approaches for signature schemes. In [3] a media authentication scheme is called secure against forgeability if for every adversary it is infeasible to produce an object $O$ and its protected version $\bar{O}$ for a given verification key. This should even hold if the adversary may ask for protected versions of other objects before. 
Our Results (Integrity). Demanding from the adversary to output a pair $(O, \bar{O})$ seems to be overly restrictive, since the authentication system should be already considered broken if an adversary merely creates an arbitrary authenticated object $\bar{O}$ (without knowing a corresponding original object $O$ ). Consider for example a governmental organization publishing satellite data $\bar{O}$ of which parts may be classified as confidential for issues of national security (contained in the encrypted $B_{O}$ part), but which should still allow public verification of authenticity. In this case, the adversary's goal could be to produce other partially protected satellite data bearing a correct signature of the governmental authority, but without any need of being able to generate a matching unprotected object. In this case, the unforgeability definition of Dittmann et al. would provide no security guarantee.

Therefore we propose a stronger definition of unforgeability, which we call strong unforgeability and which prevents attacks like the one above. To show that our definition is indeed strictly stronger than the definition of Dittmann et al., we first give a proof that strong unforgeability implies (basic) unforgeability. After that, we present an example of media authentication scheme which is secure according to the basic notion, but not according to our enhanced definition.

Before proving that the original scheme of Dittmann et al. 3] can be lifted to satisfy the notion of strong unforgeability, we need to tweak the signing process. Hopper et al. revealed in [8] that, in the original scheme, an adversary can easily find different objects that generate the same input $A_{O} \| X$ to the signing resp. verification process and thus straightforwardly constitute a forgery. We show that those attacks can be prevented by using an appropriate encoding for computing the signature, where $A_{O}$ and $X$ are clearly separated. Together with a strongly unforgeable signature scheme, this also provides a sufficient condition for a strongly unforgeable media authentication scheme.

Our Results (Secrecy). Another security aspect considered in our paper is secrecy of the original data contained in the protected object. In order to achieve a secure protection of the $B_{O}$ part, Dittmann et al. 3] propose to use a symmetric encryption scheme. Unfortunately, they neither provide any rigorous security model, nor make any conclusions about the secrecy of their scheme.

In a companion paper, Katzenbeisser and Dittmann [9] discuss a desirable secrecy requirement, resembling semantic security of encryption schemes [5] where a ciphertext should not reveal anything about the original message. In [9] the authors conclude that a similar notion for media authentication schemes "might not be possible to satisfy" because, due to the requirement of good compressibility, the protected part $B_{O}$ is typically not completely random and may statistically depend on the public part $A_{O}$. Therefore, an adversary may be able to derive some information about the encrypted part from the public part $A_{O}$. In [9] the authors thus outline an alternative (and somewhat non-standard) security definition, but remain rather informal and do not prove that the $\mathcal{M} \mathcal{A S}_{\mathrm{DKSV}}$ scheme achieves the desired level of secrecy.

Our starting point is to note that the fact that $A_{O}$ may reveal some information about $B_{O}$ does not obviate similar claims about the secrecy for the media 
authentication scheme. The reason, originating in the context of encryption, is that the precise idea of semantic security is that one should not be able to learn anything about a message $m$ from a ciphertext $X$ than what is known about $m$ anyway. For instance, if $m$ is a credit card number sent encrypted, but the card type is transmitted in clear, then the first digit is usually deducible from the type. Secrecy with respect to such side information is therefore the highest security level we can achieve and should aim for.

Adapting the notion of semantic security with side information we give a formal definition of secrecy for media authentication schemes. Our definition basically says that an authentication scheme is considered secure if whatever can be computed from a protected object $\bar{O}=\left(A_{O}, B_{\bar{O}}\right)$ could also be derived from the public part $A_{O}$ alone. We can even strengthen our notion to a more realistic scenario where the adversary is able to obtain protected and reconstructed objects of his choice. Based on the formal definition we then consider the secrecy of the media authentication scheme by Dittmann et al. and show that semantic security of the used encryption function is a sufficient condition for the authentication scheme to be semantically secure as well.

Summary. Overall, this paper here complements the work of Dittmann et al. by giving precise security models that describe the guarantees in terms of integrity and secrecy. We introduce the notion of strong unforgeability to strengthen the security against malicious modification attempts and provide the sufficient requirements for an authentication scheme to achieve this security goal. Furthermore we show that secrecy in the sense of semantic security for media authentication schemes can be defined, which is completed by proving secrecy for the construction of Dittmann et al. under reasonable assumptions about the encryption scheme.

Organization. In Section 2 we recall the definition of an invertible media authentication scheme by Dittmann et al. 3. In Section 3we introduce the scheme (or, to be precise, the framework) by Dittmann et al. and the underlying tools (watermarking, encryption and signatures). Section 4 deals with our refinement of integrity of media authentication schemes and relates the notions, whereas Section 5 covers the secrecy aspects of such schemes. We note that, following the terminology of [3], in this paper here we exclusively deal with offline media authentication. It is easy to adapt our notions and proofs to the case of online media authentication; we refer to the full version for details.

\section{Media Authentication Schemes}

An invertible media authentication scheme $(\mathcal{M A S})$, defined by Dittmann et al. 3, consists of a set of algorithms allowing to protect a media object. More precisely, an invertible $\mathcal{M} \mathcal{A S}$ is able to produce a protected media object using the algorithm PROTECT while retaining the ability to losslessly reconstruct the original media object using algorithm RECOnstRuCT. The ability for lossless reconstruction of protected media objects is typically achieved by using invertible 
watermarking schemes as introduced by Honsinger et al. [7. If a media object has been previously protected, its integrity can be unambiguously verified using algorithm VERIFY.

Usage of the above algorithms necessitates cryptographic keys for protection as well as reconstruction of media objects, which have to be kept private. However, verification of the integrity of a protected media object assumes a public verification key, thus enabling integrity checks by third parties. The generation of all necessary keys is summarized in a single algorithm GENKEY, which takes as input a security parameter and selects keys of the corresponding strength.

Definition 1. An invertible media authentication scheme is a tuple of probabilistic polynomial-time algorithms

$$
\mathcal{M A S}=(\text { GenKey, Protect, Verify, Reconstruct })
$$

with the following properties:

- GENKEy takes as input a security parameter $n$ (in unary, as $1^{n}$ ) and outputs a triple of keys $\left(K_{P}, K_{V}, K_{R}\right)$, where $K_{P}$ is the secret protection key, $K_{V}$ is the public verification key and $K_{R}$ is the secret reconstruction key.

- Protect takes as input a media object $O$ and a protection key $K_{P}$, and outputs a protected media object $\bar{O}$ or FAIL, if protection is not possible.

- VERIFY accepts as input a protected media object $\bar{O}$ and a verification key $K_{V}$, and outputs either TRUE or FALSE.

- RECONSTRUCT takes a protected media object $\bar{O}$ and a reconstruction key $K_{R}$, and outputs a media object $O$ or FAIL.

Furthermore, we require that verification and reconstruction for valid protected objects always succeeds, i.e., for any media object $O$, for all keys $\left(K_{P}, K_{V}, K_{R}\right) \leftarrow \operatorname{GenKey}\left(1^{n}\right)$ and any $\bar{O} \leftarrow \operatorname{Protect}\left(O, K_{P}\right)$, we have

$$
\begin{array}{r}
\operatorname{Pr}\left[\operatorname{Verify}\left(\bar{O}, K_{V}\right)=\mathrm{TRUE} \mid \bar{O} \neq \mathrm{FAIL}\right]=1, \\
\operatorname{Pr}\left[\operatorname{Reconstruct}\left(\bar{O}, K_{R}\right)=O \mid \bar{O} \neq \mathrm{FAIL}\right]=1 .
\end{array}
$$

\section{The DKSV Media Authentication Scheme}

In this section we first recall the basic ingredients of the media authentication scheme by Dittmann et al. 3], before presenting the actual $\mathcal{M} \mathcal{A S}_{\mathrm{DKSV}}$ scheme.

\subsection{Tools}

Recall that the basic idea of the $\mathcal{M A S}_{\text {DKSV }}$ scheme is to divide the object $O$ into a public part $A_{O}$ and a part $B_{O}$ which should be protected. This splitting (and its inverse process) are performed via an invertible watermarking scheme, as described in this Section. The $B_{O}$ part is then compressed, encrypted and signed. Encryption and Signatures are therefore described formally afterwards. 
Watermarking. Watermarking schemes are an alternative to the concept of cryptographic signatures, designed specifically to embed authentication and integrity data within media objects, thus eliminating the need for separate storage. Invertible watermarking schemes are often a special case of fragile watermarks [10] and have been introduced by Honsinger et al. [7] to address the need to re-obtain the original media object. Fridrich et al. 4] later proposed a general framework for invertible watermarking schemes that uses lossless compression to allow the reversion of the embedding process. Thereby, the ability to embed data into a media object $O$ is accomplished by two polynomial-time algorithms JoIN and SePARATE 1

- Separate takes a media object $O$ as input and produces a tuple $\left(A_{O}, B_{O}\right)$ (or the output FAIL),

- JoIn takes a pair $\left(A_{O}^{\prime}, B_{O}^{\prime}\right)$ as input and returns a media object $O^{\prime}$ (or the output FAIL).

If the following equalities hold, $\operatorname{Join}(\operatorname{Separate}(O))=O($ given $\operatorname{Separate}(O)$ $\neq$ FAIL $)$ for any object $O$, and $\operatorname{Separate}\left(\operatorname{JoIN}\left(A_{O}, B_{O}\right)\right)=\left(A_{O}, B_{O}\right)$ (given that $\left.\operatorname{JoIN}\left(A_{O}, B_{O}\right) \neq \mathrm{FAIL}\right)$ for all $A_{O}, B_{O}$, then we call the pair (JoIn, SEPARATE) an invertible watermarking scheme.

Note that the completeness condition above also provides some sort of collision-resistance for the SEPARATE algorithm. Namely, for any objects $O \neq O^{\prime}$ with $\operatorname{Separate}(O) \neq \mathrm{FAIL}, \operatorname{Separate}\left(O^{\prime}\right) \neq$ FAIL we must have $\operatorname{Separate}(O) \neq \operatorname{Separate}\left(O^{\prime}\right)$. Otherwise, if Separate returned the same output for some $O \neq O^{\prime}$, then JoIN would sometimes fail to recover the right object $O$ or $O^{\prime}$ from these identical outputs. The analogous argument applies to JoIN. We note that we could also use a relaxed version in which "bad" objects $O \neq O^{\prime}$ may exist, but then they are hard to find in reasonable time (similar to collision-resistance of hash functions). Our results remain valid under this relaxed version.

Encryption. A symmetric encryption scheme $\mathcal{E}=($ GenEnC, EnC, DEC) consists of three probabilistic polynomial-time algorithms, where algorithm GENENC on input $1^{n}$ generates a key $K_{E}$, algorithm ENC on input $K_{E}$ and message $m \in\{0,1\}^{*}$ outputs a ciphertext $X$, and algorithm DEC also takes $K_{E}$ and a ciphertext $X$ and returns $m \in\{0,1\}^{*}$ or FAIL. Furthermore, for all keys $K_{E}$ produced by $\operatorname{GenEnC}\left(1^{n}\right)$, all messages $m \in\{0,1\}^{*}$ and ciphertexts $X \leftarrow \operatorname{EnC}\left(K_{E}, m\right)$, we have $m=\operatorname{DEC}\left(K_{E}, X\right)$.

As for security of encryption schemes we follow the idea of semantic security, as defined by Goldwasser and Micali [5]. Informally, the idea of semantic security for encryption schemes is that any information $f_{\text {enc }}(m)$ an efficient adversary could learn about a message $m$ from a ciphertext $X$ could also be computed efficiently without $X$. All this holds of course relative to any side information

${ }^{1}$ These algorithms are often defined to be initialized with a watermarking key $K_{W}$. Here we presume for simplicity that this key is "hardwired" into the description of the algorithms, or that the key is available to all parties as a system parameter. The key $K_{W}$ may also contain randomness for both algorithms (if required). 
about $m$. This extra knowledge about $m$ is typically formalized by having some side information hist $_{m}$ about the message $m$.

For notational convenience we denote by $\left(m\right.$, hist $\left._{m}\right) \leftarrow(\mathcal{M}$, hist enc $)\left(1^{n}\right)$ the joint sampling process in which the message $m$ is picked according to distribution $\mathcal{M}\left(1^{n}\right)$ and, at the same time, side information hist $m$ is generated according to algorithm hist $_{\mathrm{enc}}\left(1^{n}\right)$. Note that in this process both algorithms $\mathcal{M}$ and hist enc may share state.

Definition 2. A symmetric encryption scheme $\mathcal{E}=$ (GenEnC, EnC, DeC) is called semantically secure (with respect to side information hist enc $_{\text {) }}$ if for every probabilistic polynomial-time algorithm $\mathcal{A}_{\text {enc }}$ there is a probabilistic polynomialtime algorithms $\mathcal{S}_{\text {enc }}$, the simulator, such that for every polynomial-time distribution $\mathcal{M}$ and any function $f_{\text {enc }}$ the difference

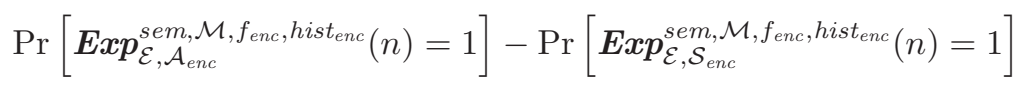

is negligible, where

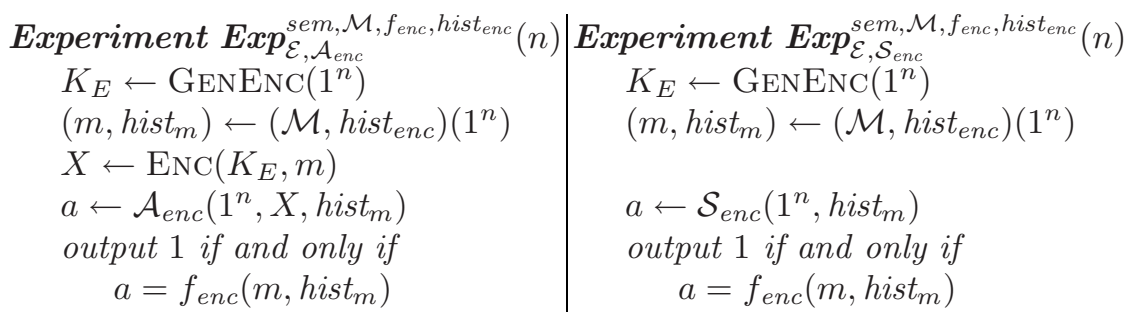

We note that Dittmann et al. 3] do not make any security claim about the underlying encryption scheme in their $\mathcal{M} \mathcal{A S}$. See also the discussion in Section5, Finally, we remark that semantic security (with respect to any side information) is a very common property of modern encryption schemes, and is usually met by all practical and theoretical solutions (cf. [6]).

Signature Schemes. A signature scheme $\mathcal{S}=$ (GEnSign, Sign, SigVERIfy) consists of probabilistic polynomial-time algorithms such that GENSIGN on input $1^{n}$ generates a key pair $\left(K_{V S}, K_{S S}\right) \leftarrow \operatorname{GENSIgN}\left(1^{n}\right)$, algorithm Sign for input $K_{S S}$ and a message $m \in\{0,1\}^{*}$ outputs a signature $s \leftarrow \operatorname{SigN}\left(K_{S S}, m\right)$, and algorithm SIGVERIFY for input $K_{V S}, m$ and $s$ returns a decision $d \leftarrow$ $\operatorname{SigVERIFY}\left(K_{V S}, m, s\right)$ which is either TRUE or FALSE. Additionally, for all security parameters $n$, all keys $\left(K_{V S}, K_{S S}\right) \leftarrow \operatorname{GenSign}\left(1^{n}\right)$, all messages $m \in$ $\{0,1\}^{*}$ and all signatures $s \leftarrow \operatorname{Sign}\left(K_{S S}, m\right)$ it holds $\operatorname{SigVERIFy}\left(K_{V S}, m, s\right)=$ TRUE.

Below we define a strong notion of security for signature schemes, called strong unforgeability, which supersedes the common notion of unforgeability for signatures (cf. 6]). Roughly, strong unforgeability also prevents the adversary from producing new signatures for previously signed messages (even if the adversary can see other signatures for chosen message through a signature oracle $\left.\operatorname{SigN}\left(K_{S S}, \cdot\right)\right)$ : 
Definition 3. A signature scheme $\mathcal{S}=$ (GenSign, Sign, SigVerify) is called strongly unforgeable if for any probabilistic polynomial-time algorithm $\mathcal{A}_{\text {sig }}$,

$$
\operatorname{Pr}\left[\boldsymbol{E x p}_{\mathcal{S}, \mathcal{A}_{s i g}}^{S t U n f}(n)=1\right]
$$

is negligible, where

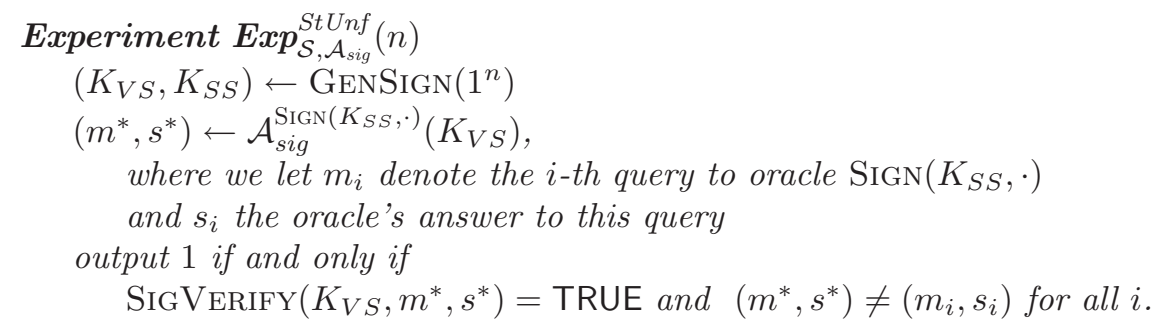

Note that in the regular notion of unforgeability we strengthen the requirement on $\left(m^{*}, s^{*}\right)$ in the experiment above, and demand that $m^{*} \neq m_{i}$ for all $i$ (such that finding another signature $s^{*}$ to a given pair $m_{i}, s_{i}$ is no longer considered a successful attack). In particular, if a scheme is strongly unforgeable, then it is also unforgeable in the basic sense. Yet, it is also easy to construct an unforgeable signature scheme which does not achieve the stronger notion, e.g., if for each signature the signing algorithm appends a redundant bit which the verification algorithm simply ignores.

Efficient strongly unforgeable schemes exist under various assumptions, e.g., [1,2]. Existentially they can be derived from any one-way function (cf. 6]) and are thus based on the same complexity assumption as signature schemes which are unforgeable in the ordinary sense.

\subsection{The $\mathcal{M} \mathcal{A} \mathcal{S}_{\mathrm{DKSV}}$ Scheme}

With the tools of the previous sections we can now recapture the $\mathcal{M} \mathcal{A} \mathcal{S}_{\mathrm{DKSV}}$ scheme. To protect a media object $O$ the $\mathcal{M A S}_{\text {DKSV }}$ scheme first uses the watermarking scheme to determine the parts $A_{O}$ and $B_{O}$. Then the $B_{O}$ part is first compressed to $C_{O}$ and together with a hash value $H(O)$ of the object, encrypted to a ciphertext $X$ 2 The resulting ciphertext and the public part $A_{O}$ of the original media object $O$ are signed together with the signature algorithm, $s \leftarrow \operatorname{Sign}\left(K_{S S},\left(A_{O}, X\right)\right)$. The values $X$ and $s$ are finally joined with $A_{O}$ into a single media object $\bar{O}$.

The integrity of a protected object $\bar{O}$ can be verified by anyone by recovering $A_{O}, X, s$ from the protected object and verifying the signature $s$ for $\left(A_{O}, X\right)$. This can be done without decrypting $X$ and recovering $B_{O}$. Reconstruction

${ }^{2}$ The role of $H(O)$ concerning the security of the scheme remains somewhat unclear, i.e., Dittmann et al. 3 never specify any security requirements on $H$. It appears that security-wise $H$ does not serve any purpose. We include $H$ here only for sake of completentess; the reader may simply think of $H$ as the function with empty output. 
then can easily be achieved by first verifying $\bar{O}$ and then decrypting with $K_{E}$. After uncompressing $C_{O}$ to $B_{O}^{\prime}$ algorithm JoIn can be applied to $\left(A_{O}, B_{O}^{\prime}\right)$. The resulting object $O^{\prime}$ is hashed to $H\left(O^{\prime}\right)$ which is compared to the embedded hash. If this is successful the restored object is returned as $O$, otherwise the reconstruction algorithm fails.

We note that, in the original scheme, Dittmann et al. use the signature algorithm to sign the concatenation $A_{O} \| X$ of the values $A_{O}$ and $X$. But this introduces a weaknesses which the attack by Hopper et al. 8] exploits. Here we therefore tweak the signature process by signing $\left(A_{O}, X\right)$ instead, with the usual meaning that this string $\left(A_{O}, X\right)$ contains a separator between the two values. For instance, we can encode the bit length of $A_{O}$ into a starting block of fixed length (say, into the first $n$ bits for security parameter $n$ ) and then append $A_{O} \| X$. Other choices are possible, of course.

Construction 1 (DKSV-MAS). Let (JoIn, SEPARATE) be an invertible watermarking scheme, $\mathcal{E}$ be a symmetric encryption scheme and $\mathcal{S}$ be a signature scheme. Let (COMPRESS, UNCOMPRESS) be a lossless compression scheme and $H$ be some function (with fixed output length). Then the DKSV media authentication scheme $\mathcal{M A S}_{D K S V}$ is defined by the following algorithms:

- Algorithm GENKEY on input $1^{n}$ runs the key generation algorithms of the signature scheme and the encryption scheme, $\left(K_{S S}, K_{V S}\right) \leftarrow \operatorname{GEnSign}\left(1^{n}\right)$ and $K_{E} \leftarrow$ GenEnC $\left(1^{n}\right)$, and outputs $K_{V}=K_{V S}, K_{R}=\left(K_{V S}, K_{E}\right)$ and $K_{P}=\left(K_{S S}, K_{E}\right)$.

- Algorithm PROTECT on input $K_{P}$ and object $O$ first splits the object by computing $\left(A_{O}, B_{O}\right) \leftarrow \operatorname{Separate}(O)$, then compresses $C_{O} \leftarrow \operatorname{Compress}\left(B_{O}\right)$ and computes a ciphertext $X \leftarrow \operatorname{ENC}\left(K_{E}, C_{O} \| H(O)\right)$. It computes a signature $s \leftarrow \operatorname{SigN}\left(K_{S S},\left(A_{O}, X\right)\right)$ and joins the signature together with $A_{O}$ and $X$ into the protected object $\bar{O} \leftarrow \operatorname{JoIN}\left(A_{O},(X, s)\right)$. It outputs $\bar{O}$ (or FAIL if any of the deployed algorithms returns FAIL).

- Algorithm VERIFY on input $K_{V}$ and a protected object $\bar{O}$ splits the object into $\left(A_{O},(X, s)\right) \leftarrow \operatorname{SEPARATE}(\bar{O})$ and returns the output of the signature verification algorithm for these data, $\operatorname{SigVERIFY}\left(K_{V S},\left(A_{O}, X\right), s\right)$ (which equals FAIL in the special case that SEPARATE returned FAIL before).

- Algorithm ReCOnstruct takes as input $K_{R}$ and a protected object $\bar{O}$, and only continues reconstruction if verification of $\bar{O}$ works. If so, then it recovers $\left(A_{O},(X, s)\right) \leftarrow$ Separate $(\bar{O})$ and decrypts $X$ to $C_{O} \| h$ and re-computes $B_{O}=\operatorname{Uncompress}\left(C_{O}\right)$ and $O \leftarrow \operatorname{JoIN}\left(A_{O}, B_{O}\right)$. If $H(O)=h$ then it outputs $O$; in any other case the algorithm returns FAIL.

\section{Integrity of Media Authentication Schemes}

In this section we address integrity protection of media authentication schemes. We first review the definition of Dittmann et al. 3] about unforgeability of 
$\mathcal{M A S} 3$ and then present our improved security guarantee, denoted by strong unforgeability. We show that strong unforgeability is strictly stronger than the notion of Dittmann et al., and finally prove that the $\mathcal{M} \mathcal{A} \mathcal{S}_{\text {DKSV }}$ scheme achieves the stronger notion if instantiated with the right primitives.

\subsection{Definitions}

The original unforgeability requirement of Dittmann et al. 3 demands that, without the protection key, it is infeasible to find an object $O$ and its protected version $\bar{O}$, even after having seen other protected objects:

Definition 4. Let $\mathcal{M A S}=($ GenKey, Protect, Verify, Reconstruct $)$ be an invertible media authentication scheme. It is called unforgeable if for every probabilistic polynomial-time algorithm $\mathcal{A}_{D K S V}$ the value

$$
\operatorname{Pr}\left[\boldsymbol{E x p}_{\mathcal{M} \mathcal{A} \text { S }, \mathcal{A}_{D K S V}}^{\operatorname{mas}}(n)=1\right]
$$

is negligible, where

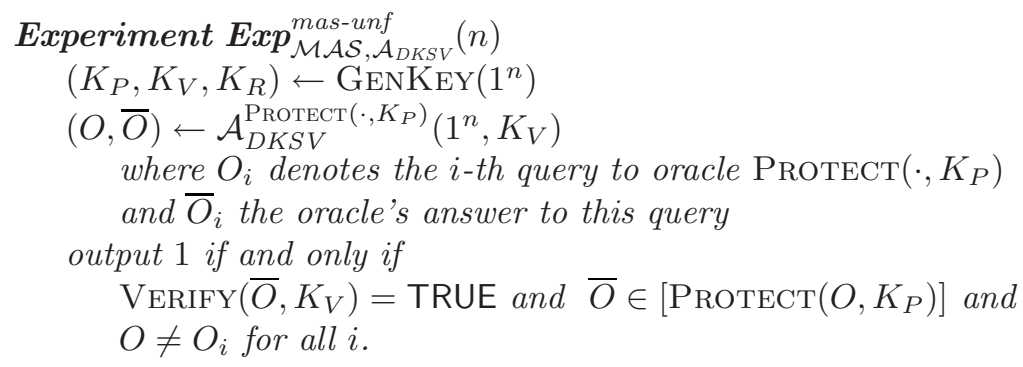

We note that Dittmann et al. 3. claim their scheme to be secure under this definition. However, as mentioned before, Hopper et al. 8] point out a gap in this proof, exploiting a weak encoding for the signing algorithm. Patching the signature and verification process as described in Construction 1 gives a version which is indeed secure according to this definition here (if the signature scheme achieves basic unforgeability). This can be easily inferred from the security proof for our stronger notion in the next section, and we therefore omit a formal proof for this simpler fact.

Our first definitional strengthening concerns the adversary's task to find a protected object $\bar{O}$ together with its original counter part $O$. Recall the satellite data example from the introduction, where the adversary's goal is only to produce another valid protected object without knowing a matching object in clear. Then the previous definition would provide no security guarantee in this case. In fact, as we will discuss later, there are even schemes satisfying the unforgeability

${ }^{3}$ Dittmann et al. call the property in their paper "security against existential unforgeability" but, for sake of better distinction with other security notions such as secrecy, we rename the property here to "unforgeability". 
notion above but which fail to meet the stronger requirement in the example. In our refinement below we therefore reduce the requirement on the adversary's output and merely demand that the attacker outputs a new protected object $\bar{O}$.

The other strengthening refers to availability of other components of a system. Since the algorithms may operate in a highly interactive setting, we follow the conservative approach in cryptography and allow our algorithm $\mathcal{A}_{\text {strong }}$ to also communicate with a RECONSTRUCT oracle, enabling him to reconstruct objects of his choice. Note that verification can be carried out locally by the adversary with the help of the public key anyway. With these two refinements we obtain the following definition:

Definition 5. Let $\mathcal{M} \mathcal{A S}=($ GenKey, Protect, Verify, Reconstruct $)$ be an invertible media authentication scheme. It is called strongly unforgeable if for every probabilistic polynomial-time algorithm $\mathcal{A}_{\text {strong }}$ the value

$$
\operatorname{Pr}\left[\boldsymbol{E x p}_{\mathcal{M A S}, \mathcal{A}_{\text {strong }}^{\text {mas-stunf }}}(n)=1\right]
$$

is negligible, where

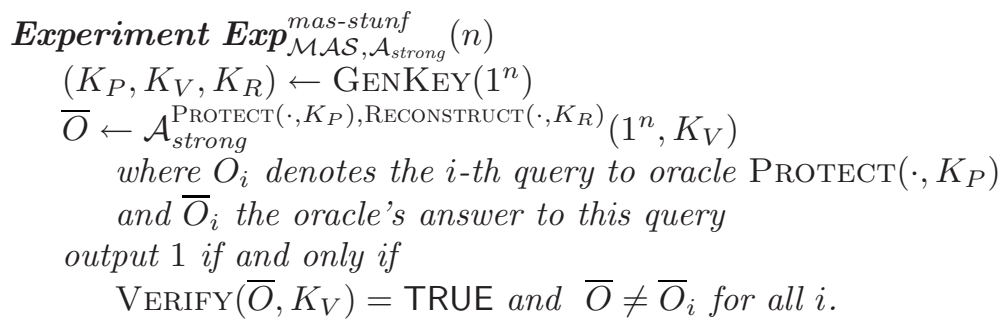

\subsection{On the Relationship of the Notions}

In this section we show that security according to our definition of strong unforgeability is strictly stronger than the one for the definition by Dittmann et al. This is done in two steps. First we will show that our definition implies the definition of Dittmann et al. After that, we provide two examples of schemes which are secure according to the basic notion but not to the enhanced definition (one example is omitted from this version here). We remark that the separating examples even hold if we augment the DKSV definition by giving $\mathcal{A}_{\mathrm{DKSV}}$ access to a ReCOnstruct oracle. This difference merely stems from the fact that $\mathcal{A}_{\mathrm{DKSV}}$ has to output a pair $(O, \bar{O})$, compared to $\bar{O}$ as in our definition.

Proposition 1. If an invertible $\mathcal{M A S}$ scheme is strongly unforgeable then it is also unforgeable.

The proof is omitted for space reasons. We next give a separating example for the patched $\mathcal{M} \mathcal{A S}_{\mathrm{DKSV}}$ framework where we assume that the signature scheme is not strongly unforgeable, i.e., where one can easily transform a signature $s$ to a message $m$ into another valid signature $s^{*} \neq s$. With this instantiation choice 
there exists a successful attack against the strong unforgeability, but which does not constitute a break against basic unforgeability.

The adversary against the strong unforgeability calls the PROTECT oracle only once about an object $O$ to derive a protected object $\bar{O}=\operatorname{Join}\left(A_{\bar{O}},(X, s)\right)$. The attacker next runs $\operatorname{Separate}(\bar{O})$ to obtain $A_{\bar{O}}=A_{O}$ and $(X, s)$. Since the signature scheme is not strongly unforgeable the attacker can now compute another valid signature $s^{*} \neq s$ for $\left(A_{O}, X\right)$. He finally outputs $\bar{O}^{*}=\operatorname{Join}\left(A_{O},\left(X, s^{*}\right)\right)$ as the forgery attempt.

The attack succeeds according to the strong unforgeability, because $s^{*} \neq s$ and thus $\bar{O}^{*}$ was never received from the PROTECT oracle before, and VERIFY evaluates to TRUE. In the DKSV definition of an attack, however, an attacker must output $(O, \bar{O})$. So in our case, prepending $O$ to $\bar{O}^{*}$ would not constitute a successful attack as $O$ has been sent to the PROTECT oracle before. In fact, it is easy to see from our proof in the next section that any attacker fails according to the DKSV definition if the underlying signature scheme achieves basic unforgeability.

\subsection{Strong Unforgeability of the $\mathcal{M} \mathcal{A} \mathcal{S}_{\mathrm{DKSV}}$-Scheme}

We next prove that the $\mathcal{M} \mathcal{A S}_{\mathrm{DKSV}}$ scheme achieves strong unforgeability if the underlying signature scheme is strong enough. Note again that this statement necessitates the patch of the signature and verification algorithm; else the attack by Hopper er al. would still apply.

Theorem 2 (Strong Unforgeability). If the signature scheme $\mathcal{S}$ is strongly unforgeable then the $\mathcal{M A S}_{D K S V}$ media authentication scheme in Construction 1 is strongly unforgeable.

Proof. If there would be a successful attacker $\mathcal{A}_{\text {strong }}$ on the $\mathcal{M} \mathcal{A S}_{\mathrm{DKSV}}$ according to our strong definition, then by using the prerequisites we could use this attacker to construct a successful attacker $\mathcal{A}_{\text {sig }}$ against the strong unforgeability of the deployed signature scheme. In the following we will show the construction of such an attacker $\mathcal{A}_{\text {sig }}$.

The attacker $\mathcal{A}_{\text {sig }}$ on the signature scheme gets the signature public key $K_{V S}$ as input. He chooses an encryption key $K_{E}$ and passes the key $K_{V}=K_{V S}$ to $\mathcal{A}_{\text {strong }}$ to start a black-box simulation. In this simulation of $\mathcal{A}_{\text {strong }}$, adversary $\mathcal{A}_{\text {sig }}$ can easily answer queries of $\mathcal{A}_{\text {strong }}$ to oracle RECONSTRUCT with the help of the key $K_{R}=\left(K_{E}, K_{V S}\right)$. For any query $O_{i}$ of $\mathcal{A}_{\text {strong }}$ to the Protect oracle, $\mathcal{A}_{\text {sig }}$ calculates $\left(A_{O_{i}}, B_{O_{i}}\right)=\operatorname{Separate}\left(O_{i}\right), C_{O_{i}}=\operatorname{Compress}\left(B_{O_{i}}\right)$ and $X_{i} \leftarrow \operatorname{ENC}\left(K_{E}, C_{O_{i}} \| H\left(O_{i}\right)\right)$. If any of the algorithms returns FAIL then $\mathcal{A}_{\text {sig }}$ immediately returns FAIL to $\mathcal{A}_{\text {strong, }}$, else $\mathcal{A}_{\text {sig }}$ passes $m_{i}=\left(A_{O_{i}}, X_{i}\right)$ to his SIGNoracle to get a signature $s_{i}$. Thereafter he returns $\bar{O}_{i}=\operatorname{JoIN}\left(A_{O_{i}},\left(X_{i}, s_{i}\right)\right)$ to attacker $\mathcal{A}_{\text {strong }}$. Once $\mathcal{A}_{\text {strong }}$ outputs a protected object $\bar{O}$ and stops, adversary $\mathcal{A}_{\text {sig }}$ runs Separate on $\bar{O}$ to obtain $A_{O}$ and $(X, s)$. Now $\mathcal{A}_{\text {sig }}$ outputs $m^{*}=$ $\left(A_{O}, X\right)$ and $s^{*}=s$.

It is obvious that $\mathcal{A}_{\text {sig }}$ perfectly mimics the PROTECT oracle as well as the ReConstruct oracle in $\mathcal{A}_{\text {strong }}$ 's emulation. It remains to show that $\mathcal{A}_{\text {sig }}$ 
succeeds in his attack whenever $\mathcal{A}_{\text {strong }}$ wins. If $\mathcal{A}_{\text {strong's output } \bar{O} \text { satisfies }}$ $\operatorname{Verify}\left(\bar{O}, K_{V}\right)=$ TRUE then in particular $\operatorname{SigVERIFY}\left(K_{V S}, m^{*}, s^{*}\right)$ for $\mathcal{A}_{\text {sig' }}$ 's output will also be TRUE and $\operatorname{Separate}(\bar{O})=\left(A_{O},(X, s)\right) \neq$ FAIL. Furthermore $\bar{O} \neq \bar{O}_{i}$ for all $i$.

We have to show that the pair $\left(m^{*}, s^{*}\right)=\left(\left(A_{O}, X\right), s\right)$ has not appeared in $\mathcal{A}_{\text {sig }}$ 's interactions with the signature oracle. This is clearly true if, in the $i$-th request, $\mathcal{A}_{\text {sig }}$ returned $s_{i}=$ FAIL before even querying the signature oracle, namely, if separation, compression or encryption failed. If, on the other hand, $\bar{O}_{i}=$ FAIL for the $i$-th interaction, because the final JOIN in the simulation of the protection query returned FAIL, but a message $m_{i}=\left(A_{O_{i}}, X_{i}\right)$ was still signed with $s_{i}$, then we must have $\left(m^{*}, s^{*}\right) \neq\left(m_{i}, s_{i}\right)$. Else, for equality $\left(m^{*}, s^{*}\right)=\left(m_{i}, s_{i}\right)$ we would have FAIL $=\operatorname{JoIn}\left(A_{O_{i}},\left(X_{i}, s_{i}\right)\right)=\operatorname{Join}\left(A_{O},(X, s)\right)=\operatorname{Join}(\operatorname{Separate}(\bar{O}))$ for $\operatorname{SePARATE}(\bar{O}) \neq$ FAIL, contradicting the completeness of the watermarking scheme. Finally, if $\bar{O}_{i} \neq$ FAIL, then because $\bar{O} \neq \bar{O}_{i}$ and the SePARATE-function is collision-resistant (see Section 3.1) we have $\left(A_{O},(X, s)\right) \neq\left(A_{O_{i}},\left(X_{i}, s_{i}\right)\right)$.

Hence, if attacker $\mathcal{A}_{\text {strong }}$ on the media authentication scheme is successful, attacker $\mathcal{A}_{\text {Sig }}$ will also succeed with the same probability, because $\left(m^{*}, s^{*}\right)$ was never received from the Sign-oracle and $\operatorname{SigVERIFY}\left(K_{V S}, m^{*}, s^{*}\right)=$ TRUE.

\section{Secrecy of Media Authentication Schemes}

Recall that the scheme by Dittmann et al. 3. introduces an encryption scheme in order to protect the $B_{O}$-part of an object $O$. However, in their paper they do not provide any claim about the secrecy under reasonable conditions about the encryption scheme, not to mention a rigorous security model. In a companion paper, though, Katzenbeisser and Dittmann 9] discuss a desirable secrecy requirement, resembling semantic security of encryption schemes (as defined in Section [3.1). Yet, their proposal advocates a somewhat elliptical mixture between semantic security and indistinguishability of encryption schemes (cf. [6]), and remains rather sketchy. It also remains unclear if, or under which conditions, the $\mathcal{M} \mathcal{A} \mathcal{S}_{\mathrm{DKSV}}$ scheme meets this goal.

Recall that the idea behind semantic security of an encryption scheme was that anything an efficient adversary could learn about a message $m$ from a ciphertext $X$ could also be computed efficiently without $X$. Here we discuss that, by using appropriate notions of secrecy with side information, we can indeed define secrecy for media authentication schemes in the sense of semantic security. Our definition basically says that an $\mathcal{M} \mathcal{A S}$ provides secrecy if whatever one can compute from a protected object $\bar{O}$ (including the public part $A_{O}$ ) could also be derived from $A_{O}$ alone 4 We then continue to show that semantic security of the encryption function (with respect to side information) also guarantees secrecy of the $\mathcal{M} \mathcal{A S}_{\mathrm{DKSV}}$ scheme.

\footnotetext{
${ }^{4}$ As usual, the adversary may have even further knowledge about (parts of) $B_{O}$ (or other information about the system) and the requirement then is that the adversary cannot deduce anything beyond this additional knowledge and $A_{O}$.
} 


\subsection{Definition}

The definition below follows the one for semantic security of encryption (with respect to side information) closely. Namely, we again compare the success probability of an adversary predicting some information $f_{\mathrm{MAS}}(O)$ of an object $O$ from the protected version $\bar{O}$ (and hist $_{O}$ ) with the prediction success of a simulator given only hist $_{O}$. For a secure $\mathcal{M A S}$ these probabilities should be close.

We write $\mathcal{O}$ for the distribution of the objects and hist MAS $_{\text {for }}$ the algorithm computing the side information. For notational convenience we again denote by $\left(O\right.$, hist $\left._{O}\right) \leftarrow\left(\mathcal{O}\right.$, hist $\left._{\mathrm{MAS}}\right)\left(1^{n}\right)$ the joint sampling process, possibly sharing state between the two algorithms.

Definition 6. An invertible media authentication scheme $\mathcal{M A S}$ is called se-

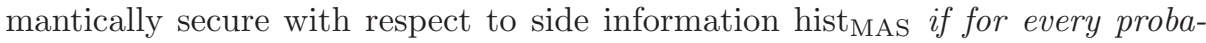
bilistic polynomial-time algorithm $\mathcal{A}_{M A S}$, there is a probabilistic polynomial-time algorithm $\mathcal{S}_{M A S}$, the simulator, such that for every polynomial-time distribution $\mathcal{O}$ of objects and for every function $f_{M A S}$, the difference

$$
\operatorname{Pr}\left[\boldsymbol{E x p}_{\mathcal{M A S}, \mathcal{A}_{M A S}^{\text {mas-sem, }}, f_{M A S}, \text { hist }_{M A S}}(n)=1\right]-\operatorname{Pr}\left[\boldsymbol{E x p}_{\mathcal{M A S}, \mathcal{S}_{M A S}}^{\text {mas-sem }, \mathcal{O}, f_{M A S}, \text { hist }_{M A S}}(n)=1\right]
$$

is negligible, where

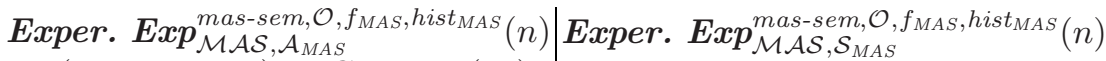

$$
\begin{aligned}
& \left(K_{P}, K_{V}, K_{R}\right) \leftarrow \operatorname{GenKey}\left(1^{n}\right) \quad\left(K_{P}, K_{V}, K_{R}\right) \leftarrow \operatorname{GenKey}\left(1^{n}\right) \\
& \left(O, \text { hist }_{O}\right) \leftarrow\left(\mathcal{O}, \text { hist }_{M A S}\right)\left(1^{n}\right) \quad\left(O, \text { hist }_{O}\right) \leftarrow\left(\mathcal{O}, \text { hist }_{M A S}\right)\left(1^{n}\right) \\
& \bar{O} \leftarrow \operatorname{Protect}\left(K_{P}, O\right) \\
& a \leftarrow \mathcal{A}_{M A S}\left(K_{V}, \bar{O}, \text { hist }_{O}\right) \\
& \text { output } 1 \text { if and only if } \\
& a=f_{M A S}\left(O, \text { hist }_{O}\right) \\
& a \leftarrow \mathcal{S}_{M A S}\left(K_{V}, \text { hist }_{O}\right) \\
& \text { output } 1 \text { if and only if } \\
& a=f_{M A S}\left(O, \text { histo }_{O}\right)
\end{aligned}
$$

We remark that we can even strengthen the notion above by granting $\mathcal{A}_{\text {MAS }}$ access to oracles $\operatorname{Protect}\left(\cdot, K_{P}\right)$ and $\operatorname{Reconstruct}\left(\cdot, K_{R}\right)$ (with the restriction that the adversary never queries the reconstruct oracle about the challenge $\bar{O}$, enabling a trivial attack otherwise). Assuming chosen-plaintext security of the underlying encryption scheme (where the adversary is also allowed to see ciphertexts of arbitrary messages via an oracle $\operatorname{ENC}\left(K_{E}, \cdot\right)$ ), our result also holds under this more advanced attack model, as we will discuss in the full version. Interestingly, the proof for this extension also takes advantage of our notion of strong unforgeability.

\subsection{Secrecy of the $\mathcal{M} \mathcal{A S}_{\mathrm{DKSV}}$-Scheme}

The following theorem shows that semantic security of the encryption scheme carries over to the secrecy of the $\mathcal{M} \mathcal{A S}_{\text {DKSV }}$ scheme:

Theorem 3. Let hist ${ }_{M A S}\left(1^{n}\right)$ be the function which takes an object $O$ and outputs $A_{O}$ where $\left(A_{O}, B_{O}\right) \leftarrow \operatorname{Separate}(O)$. Let $\mathcal{E}$ be a semantically secure encryption scheme (with respect to side information hist enc $=$ hist $_{M A S}$ ). Then the 
invertible media authentication scheme $\mathcal{M A S}_{D K S V}$ in Construction 1 is semantically secure with respect to side information hist $_{M A S}$.

The proof is by contradiction, transforming an allegedly successful adversary on the secrecy of the media authentication scheme into a successful attack against the encryption scheme. The proof appears in the full version. We also note that the result still holds if hist MAS $\left(1^{n}\right)$, in addition to $A_{O}$, includes further information like $\operatorname{hist}^{\prime}\left(B_{O}\right)$ for some function hist' (as long as the encryption scheme is secure for this augmented side information).

\section{Acknowledgments}

We thank the reviewers of Information Hiding 2007 for valuable comments.

Marc Fischlin and Anja Lehmann are supported by the Emmy Noether Program Fi 940/2-1 of the German Research Foundation (DFG).

\section{References}

1. Bellare, M., Rogaway, P.: The exact security of digital signatures -How to sign with RSA and Rabin. In: Maurer, U.M. (ed.) EUROCRYPT 1996. LNCS, vol. 1070, pp. 399-416. Springer, Heidelberg (1996)

2. Boneh, D., Shen, E., Waters, B.: Strongly Unforgeable Signatures Based on Computational Diffie-Hellman. In: Yung, M., Dodis, Y., Kiayias, A., Malkin, T.G. (eds.) PKC 2006. LNCS, vol. 3958, pp. 229-240. Springer, Heidelberg (2006)

3. Dittmann, J., Katzenbeisser, S., Schallhart, C., Veith, H.: Ensuring Media Integrity on Third-Party Infrastructures. In: Proceedings of SEC 2005. 20th International Conference on Information Security, pp. 493-508. Springer, Heidelberg (2005)

4. Fridrich, J., Goljan, M., Du, R.: Lossless data embedding - new paradigm in digital watermarking. EURASIP Journal of Applied Signal Processing 2, 185-196 (2002)

5. Goldwasser, S., Micali, S.: Probabilistic Encryption. Journal of Computer and System Science 28(2), 270-299 (1984)

6. Goldreich, O.: The Foundations of Cryptography, vol. 2. Cambridge University Press, Cambridge (2004)

7. Honsinger, C.W., Jones, P., Rabbani, M., Stoffel, J.C.: Lossless recovery of an original image containing embedded data. US patent application, Docket No: $77102 / \mathrm{E} / \mathrm{D}(1999)$

8. Hopper, N., Molnar, D., Wagner, D.: From Weak to Strong Watermarking. In: Vadhan, S.P. (ed.) TCC 2007. LNCS, vol. 4392, Springer, Heidelberg (2007)

9. Katzenbeisser, S., Dittmann, J.: Malicious attacks on media authentication schemes based on invertible watermarks. In: Security, Steganography, and Watermarking of Multimedia Contents. Proceedings of SPIE, vol. 5306, pp. 838-847 (2004)

10. Yeung, M., Mintzer, F.: Invisible watermarking for image verification. Journal of Electronic Imaging 7, 578-591 (1998) 